\title{
Clinical, Laboratory and Radiologic Course and Prognosis of COVID-19 in Cancer Patients and Comparison with General Population: A Single-Center Experience
}

\author{
Erkan ERDUR', Ozgen Ahmet YILDIRIM', Kerem POYRAZ², Canan CAN ${ }^{3}$, Halil KOMEK ${ }^{3}$ \\ ${ }^{1}$ Gazi Yasargil Training and Research Hospital, Department of Internal Medicine, Division of Medical Oncology \\ ${ }^{2}$ Gazi Yasargil Training and Research Hospital Department of Radiation Oncology \\ ${ }^{3}$ Gazi Yasargil Training and Research Hospital, Department of Nuclear Medicine, Diyarbakir, TURKEY
}

\begin{abstract}
In this study, we aimed to investigate the clinical findings and follow-up data of patients with COVID-19 and had a history of cancer, and compare these data with other COVID-19 patients. We included 2349 COVID-19 inpatients between March 2020 and July 2020. 82 of these patients were found to have a cancer diagnosis (Group 1). Patients undergoing chemotherapy were assigned to group $1 \mathrm{~A}$, patients receiving non-chemotherapy treatment were assigned to group $1 \mathrm{~B}$, cancer patients without any treatment to group $1 \mathrm{C}$ and non-cancer patients to group 2. These groups were compared in terms of basic demographic characteristics, symptoms, comorbidities, laboratory and imaging findings, intensive care unit (ICU) admission rate and death. The median age of the patients in group 1 was statistically higher than group 2 (61 and 52, $p<0.001$ ). The most common cancer diagnosis was breast cancer $(n=16,19.5 \%)$. Inflammatory parameters such as CRP and D-dimer were found to be higher in patients in group 1 than group 2 ( $p=0.005$ and $p=$ 0.003 , respectively). The mortality rate of the patients in group 1 was $15.8 \%$, while the mortality rate in group 2 was $5.1 \%$ and this was statistically significantly higher $(p<0.001)$. Being in group 1 and group $1 \mathrm{~A}$ were determined as the predictive parameters for death $(r=$ $0.087, p<0.001$ and $r: 0.254, p=0.021$ respectively). Our data suggests that history of cancer and active chemotherapy treatment are independent prognostic factors for severe disease and mortality in COVID-19.
\end{abstract}

Keywords: COVID19, Cancer Patients, Retrospective case study

\section{INTRODUCTION}

COVID-19, which emerged in the city of Wuhan city in China's Hubei Province in December 2019, has shortly become a worldwide pandemic. The virus, which is a positive-strand RNA virus from Betacoronavirus genera of in the family Coronaviridae, was initially named as 2019-Ncov but was later named as severe acute respiratory syndrome coronavirus-2 (SARS-CoV-2) by the World Health Organization (WHO) in February 2020. According to WHO data, there are 16.341 .920 cases and 650.805 deaths in 216 countries as of July 2020, posing a serious health threat. ${ }^{1}$
Even though the live animal and seafood market in Wuhan city was mentioned as the point of origin for the outbreak, COVID-19 is primarily transmitted from person-to-person via droplets or contact. ${ }^{2}$ Approximately $30-40 \%$ of patients are asymptomatic, while 60 to $70 \%$ experience different presentations ranging from mild findings to severe disease that may progress to respiratory failure, multiorgan failure, septic shock, and death. ${ }^{3}$ Advanced age and underlying comorbidities are the most important prognostic indicators in the clinical course. Comorbidities that may be associated with poor disease outcome include history of cancer, 


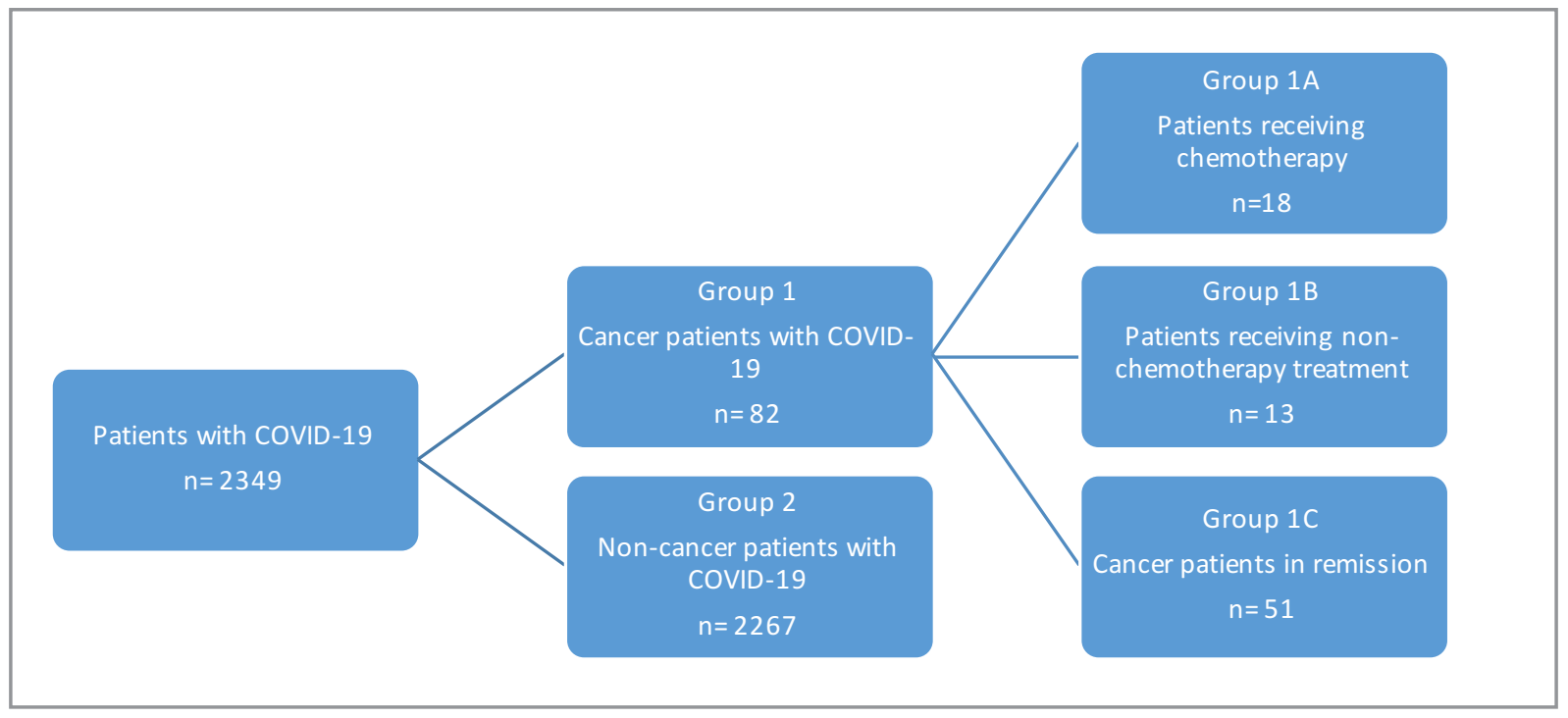

Figure 1. Study design

cardiovascular disease, diabetes, chronic pulmonary disease, chronic kidney disease, kidney transplantation, smoking, and obesity. ${ }^{4}$

WHO reported 18.1 million new cases of cancer and 9.6 million cancer-related deaths worldwide in 2018. ${ }^{5}$ Patients diagnosed with cancer are more prone to infectious diseases than the general population because of poor immune response and immunosuppressive effect of chemotherapy, radiation therapy and cancer surgery. As a matter of fact, 30day mortality being as high as $\% 18$ in cancer patients despite the $5 \%$ rate in the general population in Argentine during the 2009 influenza A (H1N1) pandemic drew attention to the importance of viral disease outbreaks in the susceptible patient population. ${ }^{6}$ There are only a limited number of studies about COVID-19 and cancer in the literature and these studies report that cancer patients are more likely to be infected with COVID-19 and have a higher mortality rate. ${ }^{7-9}$ Due to the lack of data about cancer patients infected with COVID-19, more studies about the disease course, prognostic factors, treatment approach and effects on cancer treatment in this population are required.

In this study, we aimed to investigate the clinical findings and follow-up data of patients who were admitted to our hospital for COVID-19 and had a history of cancer, and compare these data with COVID-19 patients who don't have cancer.

\section{PATIENTS and METHODS}

Two thousand three hundred and forty-nine patients who were admitted to COVID-19 quarantine clinics of our hospital between March 2020 and July 2020 were included in our study. All patients who received COVID-19 diagnosis clinically, radiographically and by reverse-transcription polymerase chain reaction (RT-PCR) technique from samples obtained from upper respiratory tract were evaluated. Patients who received a clinical or radiological diagnosis but were PCR-negative were excluded from analysis. Data related to past medical history of all patients were obtained from our hospital database and e-Nabiz database, which is the personal health database of Turkey Ministry of Health. Among our patient population, 82 patients were found to have a pathologically confirmed diagnosis of a solid organ malignancy and received either surgical treatment, chemotherapy, or radiation therapy.

Patients with a history of cancer were assigned to Group 1 and patients without a history of cancer were assigned to Group 2. Patients in Group 1 were further categorized as patients currently receiving chemotherapy (Group 1A), patients currently receiving treatment other than chemotherapy (hormone therapy, local treatment, radioactive iodine therapy, targeted therapy) (Group 1B), and patients in remission (currently not receiving any treatment) 


\begin{tabular}{|c|c|c|c|c|}
\hline & & $\begin{array}{c}\text { Group } 1(n=82) \\
n(\%)\end{array}$ & $\begin{array}{l}\text { Group } 2(n=2267) \\
n(\%)\end{array}$ & p \\
\hline \multirow[t]{2}{*}{ Sex } & Male & 38 & $1098(48.4)$ & 0.649 \\
\hline & Female & 44 & 1169 (51.6) & \\
\hline Age & & $61(26-91)$ & $52(26-91)$ & $<0.001$ \\
\hline \multirow[t]{2}{*}{ Metastatic disease } & No & $71(87.6)$ & & \\
\hline & Yes & $11(13.4)$ & & \\
\hline \multicolumn{5}{|l|}{ Comorbidities } \\
\hline \multirow[t]{2}{*}{ Hypertension } & No & $35(42.7)$ & $1104(48.7)$ & 0.335 \\
\hline & Yes & $47(57.3)$ & $1163(51.3)$ & \\
\hline \multirow[t]{2}{*}{ Diabetes } & No & $57(69.5)$ & $1773(78.2)$ & 0.146 \\
\hline & Yes & $25(30.5)$ & $494(21.8)$ & \\
\hline \multirow[t]{2}{*}{ Heart disease } & No & 60 (73.2) & $1721(75.9)$ & 0.942 \\
\hline & Yes & $22(26.8)$ & $546(24.1)$ & \\
\hline \multirow[t]{2}{*}{ Pulmonary disease } & No & $58(82.9)$ & $1922(84.8)$ & 0.100 \\
\hline & Yes & $14(17.1)$ & 345 (15.2) & \\
\hline \multirow[t]{2}{*}{ Liver disease } & No & 78 (95.1) & 2238 (98.7) & 0.068 \\
\hline & Yes & $4(4.9)$ & $29(1.3)$ & \\
\hline \multirow[t]{2}{*}{ Neurological disorder } & No & 77 (93.9) & $1972(87.0)$ & 0.062 \\
\hline & Yes & $5(6.1)$ & 295 (13.0) & \\
\hline \multirow[t]{2}{*}{ Renal disease } & No & 79 (96.3) & 2126 (93.8) & 0.565 \\
\hline & Yes & $3(3.7)$ & $141(6.2)$ & \\
\hline \multirow[t]{2}{*}{ Rheumatological disease } & No & $81(98.8)$ & 2228 (98.3) & 0.905 \\
\hline & Yes & $1(1.2)$ & $39(1.7)$ & \\
\hline \multirow[t]{2}{*}{ ICU admission } & No & $67(81.7)$ & 2047 (90.3) & 0.009 \\
\hline & Yes & 15 (18.3) & $220(9.7)$ & \\
\hline \multirow[t]{2}{*}{ Death } & Survived & $69(84.2)$ & 2151 (94.9) & $<0.001$ \\
\hline & Ex & $13(15.8)$ & $116(5.1)$ & \\
\hline \multicolumn{2}{|l|}{ Inflammatory parameters } & Median (Min-Max) & Median (Min-Max) & \\
\hline \multicolumn{2}{|l|}{ CRP } & $31.5(2-350)$ & $20.55(2-1707)$ & 0.005 \\
\hline \multicolumn{2}{|l|}{ CRPMax } & $78.8(2-350)$ & $45.95(2-724)$ & $<0.001$ \\
\hline \multicolumn{2}{|l|}{$\mathrm{LDH}$} & 258 (139-1483) & 252 (2.39-1776) & 0.418 \\
\hline \multicolumn{2}{|l|}{ LDHmax } & 317 (190-16974) & 357 (2.39-17913) & 0.105 \\
\hline \multicolumn{2}{|l|}{ DDimer } & 235 (90-9466) & $186(3-56024)$ & 0.003 \\
\hline \multicolumn{2}{|l|}{ DDimermax } & 315 (101-48923) & $248(3-64880)$ & 0.002 \\
\hline \multicolumn{2}{|l|}{ PT } & $12.4(10.5-18.8)$ & $12.5(8.7-2090)$ & 0.696 \\
\hline \multicolumn{2}{|l|}{ INR } & $1.16(0.98-1.75)$ & $1.17(0.58-155)$ & 0.627 \\
\hline \multicolumn{2}{|l|}{ Neutrophil } & $4.65(0.8-19.7)$ & $4.1(0.27-378)$ & 0.380 \\
\hline \multicolumn{2}{|l|}{ Lymphocyte } & $4.13(0.27-378)$ & $1.36(0-192)$ & 0.108 \\
\hline \multicolumn{2}{|l|}{ Hemoglobin } & $12.7(7-18.5)$ & $13.8(1.6-19.1)$ & $<0.001$ \\
\hline \multicolumn{2}{|l|}{ Platelet } & $196(28-501)$ & $203(1-855)$ & 0.424 \\
\hline NLR & & $3.34(0.09-51.93)$ & $2.94(0.01-71.21)$ & 0.131 \\
\hline
\end{tabular}

(Group 1C) (Figure 1). All groups were stratified as survivors and non-survivors. Patient age; sex; symptoms; clinical course; complete blood count parameters (white blood cell, neutrophil, lymphocyte, eosinophil, hemoglobin), C-reactive protein (CRP) level, serum lactate dehydrogenase (LDH),
D-dimer, prothrombin time (PT), activated partial thromboplastin time (aPTT), and international normalized ratio (INR) values during admission; and the highest level of CRP (CRPMax), LDH (LDHMax) and D-dimer (D-dimerMax) recorded during admission were obtained by retrospective patient 
record review and compared between groups. This study was conducted in concordance with Good Clinical Practice guidelines with a permission from the local ethics committee (No:25/09/2020-565).

\section{Statistical Analysis}

SPSS version 23.0 software was used for statistical analyses of our data. Categorical variables were represented as number and percentage, whereas continuous variables were represented as mean, standard deviation, minimum and maximum. Normality of variables were evaluated visually (histogram and probability graphics) and via analytic methods (Kolmogorov-Smirnov and Shapiro-Wilk tests). Chi-square and Fischer's exact tests were used to compare categorical variables. For normally distributed parameters, student's t test was used for comparing two variables while one-way ANOVA was preferred for more than two variables. As for non-normally distributed variables, Mann-Whitney U test was used for comparing two variables while Kruskal-Wallis test was preferred for more than two variables. Correlation between continuous variables were tested using Pearson's and Spearman's correlation analyses. $\mathrm{P}<0.05$ was considered statistically significant in all tests.

\section{RESULTS}

\section{Demographic Characteristics}

Median age of included 2349 patients was 53 years (range 26-91). Among these, 1213 patients (51.6\%) were female. Eighty-two patients (3.4\%) had cancer diagnosis (Group 1). Among these patients, 44 $(53.7 \%)$ were female and median age was 61 years (26-91).

Among 2267 patients without a history of cancer (Group 2), 1169 (51.6\%) were female. Median age was 52 years (26-91). Median age of patients in Group 1 was significantly higher than Group 2 ( $\mathrm{p}<$ 0.001). Post-hoc Bonferroni analysis revealed that mean ages of patients in Group 1A, 1B, and 1C were significantly higher than patients in Group 2 ( $p=0.003,0.011$ and $p=0.008$, respectively). Gender distribution was similar between groups ( $\mathrm{p}>$ 0.05) (Table 1).
Within Group 1, there were 16 patients $(19.5 \%)$ with breast cancer, 9 patients (11.0\%) with thyroid cancer, 8 patients $(9.8 \%)$ with lung cancer, 6 patients $(7.3 \%)$ with colon cancer, 5 patients $(6.1 \%)$ with prostate cancer, 5 patients $(6.1 \%)$ with basal cell skin carcinoma, 4 patients $(4.9 \%)$ with brain cancer, 4 patients (4.9\%) with ovarian cancer, 4 patients $(4.9 \%)$ with rectal cancer, 4 patients $(4.9 \%)$ with bladder cancer, 4 patients $(4.9 \%)$ with gastric cancer, 3 patients $(3.7 \%)$ with renal cancer, 2 patients $(2.4 \%)$ with testicular cancer, 2 patients (2.4\%) with mesothelioma, 2 patients $(2.4 \%)$ with mesenchymal malignancies, 1 patient $(1.2 \%)$ with cervical cancer, 1 patient (1.2\%) with endometrium cancer, 1 patient (1.2\%) with pancreatic cancer, and 1 patient $(1.2 \%)$ with thymoma.

Within Group 1, 18 patients (21.9\%) were undergoing active chemotherapy (Group 1A), 13 patients (15.8\%) were undergoing either hormone therapy, local treatment, radioactive iodine therapy or targeted therapy (Group 1B) and 51 patients (62.3\%) were not receiving any cancer treatment and considered in remission (Group 1C). 10 out of 18 patients $(55.6 \%)$ in Group $1 \mathrm{~A}$ and 1 out of 13 patients $(7.7 \%)$ in Group 1B had metastatic disease.

Among 51 patients (62.3\%) in Group 1C who are in remission, median duration of remission was 6 years (range: 2-13 years). Mortality rates of patients who were in remission for less than 6 years was significantly higher than patients who were in remission for more than 6 years and patients in Group 2 (p: 0.01)

All patients in Group 1 had fatigue. Sixty-seven patients $(82.3 \%)$ had fever, $70(84.8 \%)$ had coughing, $64(78.5 \%)$ had myalgia, 33 (40.5\%) had nausea and vomiting, 7 (8.9\%) had abdominal pain, and $4(5.1 \%)$ had diarrhea. Among patients in Group 2, $2086(92 \%)$ had fatigue, $1816(80.1 \%)$ had fever, $1866(82.3 \%)$ had coughing, $1800(79.4 \%)$ had myalgia, 875 (38.6\%) had dyspnea, 803 (35.4\%) had nausea and vomiting, $206(9.1 \%)$ had abdominal pain, and 109 (4.8\%) had diarrhea. There was no significant difference between two groups in terms of symptoms ( $p>0.05$ for all).

No statistically significant difference was observed between groups in terms of comorbidities including hypertension, diabetes, heart disease, neuro- 
International Journal of Hematology and Oncology

\begin{tabular}{|c|c|c|c|c|c|}
\hline & $\begin{array}{l}\text { Group 1A } \\
\text { Median (Min-Max) }\end{array}$ & $\begin{array}{l}\text { Group 1B+C } \\
\text { Median (Min-Max }\end{array}$ & $\begin{array}{l}\text { Group } 2 \\
\text { Median (Min-Max }\end{array}$ & $\mathbf{p}$ & Post hoc significance \\
\hline CRP & 86.95 (3.6-350.0) & $30.35(2-350)$ & $20.55(2-1707)$ & 0.005 & $\begin{array}{l}1 A-1 B+1 C ; p=0.049 \\
1 A-2 ; p=0.002\end{array}$ \\
\hline CRPMax & $130(17.7-350)$ & $62.45(2-350)$ & $45.95(2-724)$ & $<0.001$ & $1 A-2 ; p<0.001$ \\
\hline $\mathrm{LDH}$ & $269.5(157-1317)$ & 257 (139-1483) & 252 (2.39-1776) & 0.418 & No significant difference \\
\hline LDHmax & 324 (191-16974) & $362.5(190-4500)$ & 317 (2.39-17913) & 0.105 & No significant difference \\
\hline DDimer & 494 (132-7599) & $185(90-9466)$ & $186(3-56024)$ & 0.003 & $1 \mathrm{~A}-1 \mathrm{~B}+1 \mathrm{C} ; \mathrm{p}=0.041$ \\
\hline DDimermax & 715 (136-13825) & 294 (101-48923) & $248(3-64880)$ & 0.002 & $1 \mathrm{~B}+1 \mathrm{C}-2 ; p=0.034$ \\
\hline PT & 12.65 (10.7-18.8) & $12.4(10.5-16.6)$ & $12.5(8.7-2090)$ & 0.696 & No significant difference \\
\hline INR & $1.18(1-1.75)$ & $1.16(0.98-1.54)$ & $1.17(0.58-155)$ & 0.627 & No significant difference \\
\hline Neutrophil & $5.22(0.8-13.6)$ & $4.6(1.84-19.7)$ & $4.1(0.27-378)$ & 0.380 & No significant difference \\
\hline Lymphocyte & $0.96(0.15-43.6)$ & $1.2(0.4-3.32)$ & $1.36(0-192)$ & 0.108 & No significant difference \\
\hline Hemoglobin & $10.55(7.9-16.0)$ & $13.35(7-18.5)$ & $13.8(1.6-19.1)$ & $<0.001$ & $\begin{array}{l}1 B+1 C-1 A ; p<0.001 \\
2-1 A ; p<0.001\end{array}$ \\
\hline Platelet & $195.5(28-488)$ & $196(49-501)$ & $203(1-855)$ & 0.424 & No significant difference \\
\hline NLR & 3.57 (0.09-51.93) & 3.27 (0.93-24.62) & $2.94(0.01-713.21)$ & 0.131 & No significant difference \\
\hline
\end{tabular}

logical disorders, pulmonary disease, liver disease, renal disease and rheumatological diseases ( $\mathrm{p}>$ 0.005 for all) (Table 1).

Seventy-eight patients (94.9\% for all) in Group 1 had findings suggesting viral pneumonia in lung computerized tomography (CT) obtained at the time of admission, while in Group 2, 2130 out of 2267 patients $(94.1 \%)$ had findings suggesting viral pneumonia. The difference was not statistically significant $(\mathrm{p}=0.16)$.

\section{Comparison of Laboratory Parameters}

CRP, CRPMax , D-dimer and D-dimerMax levels of patients in Group 1 were higher than Group 2, while hemoglobin levels were significantly lower ( $p<0.05$ for all). There was no statistically significant difference between two groups in other laboratory parameters (Table 1).

Among subgroups of Group 1 (Group 1A, 1B and 1C), levels of serum LDH, D-dimer and hemoglobin were significantly different $(p=0.026$, 0.025 , and 0.003 , respectively). Post hoc analyses were performed to determine the source of this difference revealed that serum LDH levels were significantly higher in Group 1B than Group 1C, D-dimer levels were significantly higher in Group
1A than Group 1C, and hemoglobin levels were significantly higher in Group 1C than Group 1A $(\mathrm{p}<0.05$ for all $)$

Statistically significant differences were observed between cancer patients receiving chemotherapy (Group 1A), not receiving chemotherapy (Group $1 \mathrm{~B}+1 \mathrm{C}$ ) and non-cancer patients (Group 2) in terms of CRP, CRPMax, D-Dimer and D-dimerMax values $(p=0.005,<0.001,0.003$ and 0.002 , respectively). Post hoc analyses performed to determine the source of this difference revealed that serum CRP levels were significantly higher in Group 1A than Group $1 \mathrm{~B}+1 \mathrm{C}(\mathrm{p}=0.049)$ and Group $2(\mathrm{p}=$ $0.002)$, D-dimer levels were significantly higher in Group 1A than Group 1B+1C ( $\mathrm{p}=0.041)$, and $\mathrm{D}$-dimerMax levels were significantly higher in Group 1B+1C than Group 2 ( $\mathrm{p}=0.034)$ (Table 2).

Hemoglobin levels were significantly higher in Group $1 \mathrm{~B}+1 \mathrm{C}$ than Group 1A $(\mathrm{p}<0.001)$ and in Group 2 than Group 1A ( $<<0.001)$ (Table 2).

\section{Comparison of Need for Intensive Care and Mortality}

Among 82 patients in Group 1, 15 patients (18.2\%) required intensive case unit (ICU) admission due to respiratory distress. Noninvasive mechanical 
Table 3. Correlation of mortality with age, cancer history, chemotherapy and laboratory parameters

\begin{tabular}{|c|c|c|c|c|c|c|}
\hline & \multirow{2}{*}{\multicolumn{2}{|c|}{$\begin{array}{l}\text { Entire Population } \\
\text { Mortality }\end{array}$}} & \multirow{2}{*}{\multicolumn{2}{|c|}{$\frac{\text { Group1 }}{\text { Mortality }}$}} & \multirow{2}{*}{\multicolumn{2}{|c|}{$\begin{array}{l}\text { Group2 } \\
\text { Mortality }\end{array}$}} \\
\hline & & & & & & \\
\hline & $\mathbf{r}$ & $\mathbf{p}$ & $\mathbf{r}$ & $\mathbf{p}$ & $\mathbf{r}$ & $\mathbf{p}$ \\
\hline LDH (Mean: 280.69) & 0.175 & $<0.001$ & 0.162 & $<0.001$ & 0.433 & $<0.001$ \\
\hline D-dimer (Mean: 432.89) & 0.261 & $<0.001$ & 0.237 & $<0.001$ & 0.610 & $<0.001$ \\
\hline CRP (Mean: 44.45) & 0.253 & $<0.001$ & 0.244 & $<0.001$ & 0.368 & $<0.001$ \\
\hline Lymphocyte (Mean: 1.74 and below) & -0.076 & $<0.001$ & -0.079 & $<0.001$ & -0.037 & $<0.001$ \\
\hline NLR & 0.174 & $<0.001$ & 0.173 & $<0.001$ & 0.393 & $<0.001$ \\
\hline Age & 0.276 & $<0.001$ & 0.263 & $<0.001$ & 0.424 & $<0.001$ \\
\hline Cancer history & 0.087 & $<0.001$ & & & & \\
\hline Chemotherapy & 0.254 & 0.021 & & & & \\
\hline Non-chemotherapy treatment & 0.039 & 0.728 & & & & \\
\hline
\end{tabular}

ventilation (NIMV) was applied to all patients during their ICU stay, while 14 patients $(93.3 \%)$ necessitated invasive mechanical ventilation (IMV) during their course. Thirteen patients $(92.8 \%)$ who required IMV died (Table 1). Mortality rate in Group 1 was $15.8 \%$.

Among 2267 patients in Group 2, 220 patients (9.7\%) required ICU admission due to respiratory distress. NIMV was applied to 213 patients (96.8\%) during their ICU stay. Among those, 165 patients $(77.4 \%)$ necessitated invasive mechanical ventilation during their course. One hundred and sixteen patients $(70.3 \%)$ who required IMV died (Table 1). Mortality rate in Group 2 was $5.1 \%$.

The need for intensive care was significantly higher in Group 1 than Group $2(p=0.009)$. The rate of mortality in Group 1 was also significantly higher than Group $2(\mathrm{p}<0.001)$ (Table 1).

Correlation between patient characteristics and mortality was also analyzed. It was observed that mortality did not favor either genders. When Groups 1 and 2 were analyzed separately, a positive correlation between age and mortality was observed $(\mathrm{p}<0.001, \mathrm{r}=0.276)$ (Table 3). Multivariate analyses of the entire population revealed that history of cancer and receiving chemotherapy for cancer were positively correlated with mortality $(\mathrm{p}=0.021, \mathrm{r}=0.254)$. We found no significant correlation between receiving treatment other than chemotherapy for cancer or mortality $(\mathrm{p}=0.728, \mathrm{r}=$ 0.039) (Table 3).
There was a negative correlation between lymphocyte count and mortality in both Group 1 and Group 2. Neutrophil/lymphocyte ratio (NLR) had a significant positive correlation with mortality in both groups. Similarly, inflammatory parameters including serum LDH, D-dimer and CRP levels also had a significant positive correlation with mortality in both groups ( $p<0.05$ for all). No significant correlation was observed between other laboratory parameters and mortality $(\mathrm{p}>0.05$ for all) (Table 3).

\section{DISCUSSION}

In our study, we investigated the clinical, laboratory and radiologic characteristics of COVID-19 in patients with or without cancer and compared the groups to determine the prognostic significance of these characteristics. Along with general prognostic factors like age and comorbidities, we observed that having a cancer diagnosis, receiving active chemotherapy and laboratory parameters including serum LDH, CRP, D-dimer, lymphocyte count and NLR predicted severe disease course and mortality. Receiving non-chemotherapy cancer treatment had no significant correlation with mortality.

Patients with cancer are susceptible to infections diseases because of immunosuppression. ${ }^{10}$ In a study performed in the early periods of SARS$\mathrm{CoV}-2$ pandemic, cancer patients are shown to have 2.3 times greater risk for COVID-19 infection than the normal population due to immunosuppressive treatments and frequent hospital visits. ${ }^{7}$ 
In one of the first studies concerning COVID-19 and cancer, Liang et al. reported the prevalence of cancer to be $1 \%$ in 1590 patients infected with SARS-CoV-2. This rate is higher than $0.29 \%$ cancer prevalence in the Chinese population. The same study also reported that cancer patients had higher need for intensive care and ventilator and higher mortality than patients without cancer $(39 \%$ vs $8 \%$, respectively; $\mathrm{p}<0.001) .{ }^{8}$ Another Chinese study with 44672 patients showed that mortality rates in patients with or without cancer were $5.6 \%$ and $2.3 \%$, respectively, despite the low number cancer patients included. ${ }^{9}$

Many types of cancer are known to increase with advancing age. ${ }^{11}$ In our study, median age of patients in Group 1 was significantly higher than patients in Group 2, a difference applying to all subgroups of Group 1. This finding implies that patients in Group 1 are more susceptible to get infected with COVID-19 and prone to severe clinical course for advanced age as well, which is consistent with a current Chinese study. ${ }^{12}$ Even though age is an important parameter predicting mortality in COVID-19 infection, Qiubai Li et al found that cancer patients younger than 65 years had higher in-hospital mortality rates than patients older than 65 years. ${ }^{13}$ This data indicates that increased mortality in cancer patients cannot only be explained by the higher mean age in this group.

Fatigue, fever, coughing, myalgia, and gastrointestinal complaints are reported to be the most frequent symptoms in COVID-19 patients. ${ }^{14}$ In our study, the distribution of patient symptoms was also consistent with the literature. There was no difference in presenting symptoms between patients in Groups 1 and 2. Our patient groups had no significant different in terms of comorbid diseases.

There was no difference in lung CT findings at the time of hospital admission between Group 1 and Group 2. The most common findings were bilateral ground glass opacities and consolidations suggesting viral pneumonia, and this was similar to radiologic findings in the study by Chen et al. ${ }^{15}$ Even though Song et reported 14\% normal lung CT finding $\mathrm{s}^{16}$, this rate was $5 \%$ for both groups in our study.
In order to evaluate the role of elevated CRP, LDH and D-dimer levels in predicting severe disease and mortality in COVID-19 patients, we compared the results obtained during patients' hospital stay. CRP, CRPMax, D-dimer, and D-dimerMax levels of patients in Group 1 were significantly higher and hemoglobin levels were significantly lower than Group 2. These findings indicated that cancer patients had higher risk of ARDS and mortality, which was consistent with the study by Evangelos et al. ${ }^{17}$ Low hemoglobin levels were considered to be due to bone marrow suppression, anemia of chronic disease or nutritional deficiency in patients receiving chemotherapy.

Within the subgroups of Group 1, serum LDH levels were significantly higher in Group 1B than Group 1C and D-dimer levels were significantly higher in Group 1A than Group 1C. Previous studies have shown that elevated D-Dimer and LDH levels indicated poor prognosis in COVID-19 patients and cancer patients receiving any treatment had more severe disease than cancer patients who are in remission at the time of COVID-19 diagnosis. ${ }^{18,19}$

CRP levels of Group 1A were significantly higher than Group 1B+1C and Group 2, where D-dimer levels were only higher than Group $1 \mathrm{~B}+1 \mathrm{C}$. Elevated CRP and D-dimer levels are reported to predict poor prognosis in COVID-19 infection. ${ }^{20}$ Our findings suggested that patients receiving chemotherapy had more severe COVID-19 infection at diagnosis than cancer patients not receiving chemotherapy and non-cancer patients.

In concordance with the laboratory findings, $18.3 \%$ of the patients in Group 1 needed intensive care during their course while this rate was $9.7 \%$ in Group 2. Subgroup analyses revealed that patients in Groups 1A and 1B who receive any oncologic treatment required intensive care more than patients in Groups $1 \mathrm{C}$ and 2. This was also consistent with previous literature..$^{8-13}$

Mortality rate of patients in Group 1 was significantly higher than the mortality rate in Group 2 $(15.8 \%$ and $5.1 \%$, respectively; $\mathrm{p}<0.001)$. This finding supported the study by Quibai L et al. that found higher mortality rates in cancer patients. ${ }^{13}$ 
The mortality rate in cancer patients in our study was higher than the mortality rate of cancer patients in the Chinese population $(15.8 \%$ and $5.6 \%$, respectively). ${ }^{8}$ However, in the study that evaluated 355 patients who died of COVID-19 in Italy, Onder et al. reported that 72 patients $(20 \%)$ had a history of malignancy. ${ }^{21}$ Also, Vikas et al. reported $25 \%$ mortality in patients with a solid tumor. ${ }^{22}$ These may indicate that mortality may be affected by geographical conditions and differences in healthcare systems in different countries. A metanalysis found $21.1 \%$ mortality in COVID-19 patients with cancer. ${ }^{23}$

The limitations of our study were sharing experience from a single center and its retrospective nature. However, the burden of COVID-19 pandemic on healthcare systems is unprecedented and healthcare workers are expected to make life-changing decisions in a short period of time with limited experience. Therefore, predictive markers should be carefully identified in order to enlighten our approaches.

In conclusion, we have shown that history of cancer and receiving chemotherapy are independent prognostic factors for severe disease and mortality in COVID-19. Also, we observed that laboratory parameters, including CRP, LDH, NLR and D-dimer, that have been previously defined as prognostic markers for COVID-19 course predicted severe disease in these patient groups. Cancer patients should be more careful about isolation precautions, mask-wearing and social distancing practices. In the case of suspicious contact, it should be kept in mind that the disease is likely to have a severe course in this special patient group.

\section{REFERENCES}

1. World Health Organization. Director-General's remarks at the media briefing on 2019-nCoV on 11 February 2020.https:// www.who.int/dg/speeches/detail/who-director-general-sremarks-at-the-media-briefing-on-2019-ncov-on-11-february-2020 Situation Report - 190 Data as received by WHO from national authorities by 10:00 CEST, 28 July 2020.

2. World Health Organization. Novel Coronavirus (2019-nCoV) technical guidance. https://www.who.int/emergencies/diseases/novel-coronavirus-2019/technical-guidance
3. Richardson S, Hirsch J S, Narasimhan M, et al. Presenting characteristics, comorbidities, and outcomes among 5700 patients hospitalized with COVID-19 in the New York City area. JAMA 323: 2052-2059, 2020.

4. Naomi H, Peter K, Partha K, et al Risk factors for COVID19-related mortality in people with type 1 and type 2 diabetes in England: a population-based cohort study. Lancet Diabetes Endocrinol 8: 823-833, 2020.

5. Bray F, Ferlay J, Soerjomataram I, Torre LA, Jemal A. Global cancer statistics 2018: GLOBOCAN estimates of incidence and mortality worldwide for 36 cancers in 185 countries. CA Cancer J Clin 70: 394-424, 2018.

6. Dignani MC, Costantini P, Salgueira C, et al. Pandemic 2009 influenza $\mathrm{A}(\mathrm{H} 1 \mathrm{~N} 1)$ virus infection in cancer and hematopoietic stem cell transplant recipients; a multicenter observational study. F1000 Research 2015, 3: 221, 2014.

7. Yu J, Ouyang W, Chua MLK, Xie C. SARS-CoV-2 Transmission in patients with cancer at a tertiary care hospital in Wuhan, China. JAMA Oncol 6: 1108-1110, 2020.

8. Liang W, Guan W, Chen R et al. Cancer patients in SARSCoV-2 infection: A nationwide analysis in China. Lancet Oncol 21: 335-337, 2020.

9. Zhang $L, Z$ Zhu F, Xie $L$ et al. Clinical characteristics of COVID19-infected cancer patients: a retrospective case study in three hospitals within Wuhan, China. Ann Oncol 31: 894-901, 2020.

10. Kamboj M, Sepkowitz K A. Nosocomial infections in patients with cancer. Lancet Oncol. 10: 589-597, 2009.

11. Hayat M J, Howlader N, Reichman ME, et al. Cancer statistics, trends, and multiple primary cancer analyses from the Surveillance, Epidemiology, and End Results (SEER) Program. Oncologist 12: 20-37, 2007.

12. Yang $X, Y u Y, X u$ J, et al. Clinical course and outcomes of critically ill patients with SARS-CoV-2 pneumonia in Wuhan, China: a single-centered, retrospective, observational study. Lancet Respir Med 8: 475-481, 2020.

13. Li Q, Chen L, Li $Q$ et al. Cancer increases risk of in-hospital death from COVID-19 in persons $<65$ years and those not in complete remission. Leukemia 34: 2384-2391, 2020.

14. Cevik M, Bamford CGG, Ho A. COVID-19 pandemic-a focused review for clinicians. Clin Microbiol Infect. 26: 842-847, 2020.

15. Chen N, Zhou M, Dong X, et al. Epidemiological and clinical characteristics of 99 cases of 2019 novel coronavirus pneumonia in Wuhan, China: a descriptive study. Lancet 395: 507-513, 2020.

16. Song F, Shi N, Shan F, et al. Emerging 2019 Novel Coronavirus (2019-nCoV) Pneumonia. Radiology. 295: 210-217, 2020.

17. Evangelos $T$, loannis N S, Ismail E, et al. Hematological findings and complications of COVID-19 Am J Hematol 95: 834$847,2020$. 
18. Gao Y, Li T, Han M, et al. Diagnostic utility of clinical laboratory data determinations for patients with the severe COVID-19. J Med Virol 7: 791-796, 2020.

19. Brandon M H, Gaurav A, Wong J, et al. Lactate dehydrogenase levels predict coronavirus disease 2019 (COVID-19) severity and mortality: A pooled analysis. Am J Emerg Med 38: 1722-1726, 2020.

20. Muhammed K, Raveena K, Kiran P. The role of biomarkers in diagnosis of COVID-19 A systematic review. Life Sci 254: 117788, 2020.

21. Onder G, Rezza G, Brusaferro S. Case-fatality rate and characteristics of patients dying in relation to COVID-19 in Italy. JAMA 323: 1775-1776, 2020.

22. Mehta V, Goel S, Kabarriti R, et al. Case fatality rate of cancer patients with COVID-19 in a New York Hospital System. Cancer Discovery 7: 935-941, 2020.

23. ElGohary G M, Hashmi S, Styczynski J, et al. The risk and prognosis of COVID-19 infection in cancer patients: A systematic review and meta-analysis. Hematol Oncol Stem Cell Ther S1658-3876: 30122-30129, 2020.

\section{Correspondence:}

\section{Dr. Erkan ERDUR}

Gazi Yasargil Egitim ve Arastirma Hastanesi

Ic Hastaliklari Anabilim Dali, Medikal Onkoloji Bolumu

21010 Kayapinar

DIYARBAKIR / TURKEY

Tel: (+90-505) 3710886

Fax: (+90-412) 2580060

e-mail: erdurerkan@gmail.com

\section{ORCIDs:}

Erkan Erdur

Ozgen Ahmet

Kerem Poyraz

Halil Komek

Canan Can
0000-0002-9123-2688

0000-0002-4139-067X

0000-0001-7057-8893

0000-00018168-136X

0000-0002-0255-5830 